\title{
The Importance of Magnitude Information in Numerical Processing: Evidence from the SNARC Effect
}

\author{
Wim Fias, Marc Brysbaert, \\ Frank Geypens, and Géry d'Ydewalle \\ University of Leuven, Leuven, Belgium
}

\begin{abstract}
Models of the numerical cognitive system differ in the importance they attach to magnitude information in numerical processing. In this paper, the necessity of addressing a central semantic magnitude system in arabic number processing is evaluated by looking at the SNARC-effect (Dehaene, Bossini, \& Giraux, 1993). The existence of a Spatial-Numerical Association of Response Codes (small numbers are reacted to faster with the left hand, large numbers with the right hand) has been interpreted as an indication of access to the semantic system. In Experiment 1, we replicated the effect in a parity judgement task. In Experiment 2, we extended the SNARC-effect to a phoneme monitoring task, showing that magnitude information was accessed during arabic-to-verbal transcoding. Experiment 3 demonstrated that the SNARC effect in the phoneme monitoring task did not change with practice. We conclude, therefore, that number magnitude plays a more important role than is generally accepted.
\end{abstract}

Much scientific effort has recently been devoted to the understanding of numerical cognition (see especially Dehaene, 1992a). Although this has led to quite different theories, there is considerable agreement on the kind of research questions that deserve primary attention: How and in what forms are numbers represented, and how do these information stores interact in numerical processing?

Numbers can be represented in three main formats: arabic (numbers in numerical format), verbal (numbers in graphemic or phonological word format), and magnitude-related. The main point of interest-and of disagreement-concerns the functional relationship between these different

Requests for reprints should be sent to Wim Fias, Department of Psychology, University of Leuven, Tiensestraat 102, B-3000 Leuven, Belgium. E-mail: wim.fias@psy.kuleuven.ac.be

This manuscript was made possible by the Research Council of the University of Leuven (G.O.A. grant 93/1) and by the National Fund for Scientific Research (Belgium), of which Marc Brysbaert is research associate. We wish to thank Peter De Graef, Karl Verfaillie, Stanislas Dehaene, and an anonymous reviewer for useful comments and suggestions on an earlier draft of this manuscript. 
representation stores and their processing characteristics. In particular, the importance of magnitude information needs to be clarified, because magnitude is the semantic aspect of numerical processing. Each number, whatever its format, is a symbolic representation of a magnitude or quantity. Therefore, understanding the processing details of magnitude in relation to arabic and verbal representations is imperative. In addition, it has been shown that magnitude plays a role not only when it is expected from the numerical nature of the task (quantity estimation, counting, number comparison, mental arithmetic, etc.) but also in non-numerical tasks, in which no influence would be expected. For instance, it has been shown that the numerical size of digits interferes with the comparison of the physical size of the digits (Henik \& Tzelgov, 1982), and that subjects are slower to decide that two digits are not physically identical when the digits are numerically close than when they are not (Dehaene \& Akhavein, 1995).

With respect to the functional locus of magnitude information, there are two opposed views on how magnitude is related to the other representations. According to one view, access to magnitude information is necessary and obligatory before any other numerical processing can take place. This position is primarily defended by McCloskey and colleagues (McCloskey, Caramazza, \& Basili, 1985; see also McCloskey, 1992; McCloskey \& Caramazza, 1987; McCloskey, Sokol, \& Goodman, 1986). Their highly modular model assumes notation-specific (verbal or arabic) comprehension modules that translate numerical input into an abstract and amodal number code, from which calculation procedures and number knowledge are accessed. The amodal information is then translated to a notation-specific code, which is further handled by notation-specific production modules for either verbal or arabic, written or oral, output. The view that all numerical input is gated to an amodal representation is also advocated by Gallistel and Gelman (1992).

Other theories reject such a central bottleneck. This is the case for Dehaene's triple-code model (1992b), Noël and Seron's preferred entry code model (1993), and Campbell and Clark's encoding complex hypothesis (1988; see also Campbell, 1994). Dehaene's triple-code model (1992b) assumes that the three representational codes (verbal, arabic, and amodal magnitude information) each constitute the starting point for different arithmetic activities. Multi-digit operations, for instance, are assumed to start from arabic representations, number size comparisons from the amodal magnitude representations, and retrieval of arithmetic facts from the verbal representations. During processing, information can be exchanged between the systems. Thus, if a problem is presented in a non-specialised modality, the information will be transcoded to the relevant modality in which further processing takes place. On this account, access to the amodal magnitude information is necessary only for certain tasks (e.g. subitizing, estimation, approximate calculation, and comparison), while it is non-obligatory for other types of tasks (e.g. counting, arithmetical fact retrieval, etc.). 
Noël and Seron's preferred entry code model (1993) assumes a unique, idiosyncratic preferred entry to access number knowledge and calculation procedures: Some subjects have access to number knowledge from arabic representations, others from verbal representations. If a number is presented in the non-preferred modality, a direct, asemantic transcoding to the other modality is performed, from which magnitude and other semantic number knowledge is accessible.

The encoding complex hypothesis (Campbell, 1994; Campbell \& Clark, 1988 ) assigns a primary role to modality-specific processes and representations and rejects the assumption of abstract representations. Instead, the modalityspecific number codes collectively form an encoding complex that mediates numerical functions.

The second controversy in current theorising about number processing concerns the nature of the magnitude information. According to McCloskey (1992), this information is represented in a digital format with base-ten structure. A number is seen as a sum of units $\left(10^{\circ}\right)$, tens $\left(10^{1}\right)$, hundreds $\left(10^{2}\right)$, and so forth. On the other hand, Dehaene (1992b; Dehaene, Bossini, \& Giraux, 1993) proposes an analogue format, which is conceptualised as an oriented mental number-line (another conceptualisation of number magnitude in an analogue format has been proposed by Gallistel and Gelman, 1992). Part of the evidence for this representation comes from the Spatial-Numerical Association of Response Codes (SNARC) in parity judgement (Dehaene et al., 1993). In a binary response setting, it has been found that relatively small numbers are reacted to faster with the left hand than with the right hand, whereas relatively large numbers elicit faster responses with the right hand than with the left hand. This SNARC effect is thought to originate from the fact that the mental number line is oriented from left to right (in the case of left-right reading cultures), so that there is congruity between small numbers and left-side responses and between large numbers and right-side responses.

It is precisely the possible presence of a SNARC effect that is used in the experiments below to specify the nature and the necessity of magnitude information in number recognition. If one assumes that Dehaene's idea of an oriented number line is correct, and that McCloskey's, and Gallistel and Gelman's, hypothesis of a pivotal role of number magnitude holds, then one has to predict that the SNARC effect will be present in every number transcoding task. This is tested here with a task in which arabic input must be translated to a verbal output code.

It may be noted that the above view is more extreme than the current models, because of the double assumption of orientation and necessity of magnitude addressing. McCloskey, and Gallistel and Gelman, need not predict a SNARC effect for an arabic-to-verbal transcoding task because their abstract semantic number code is not supposed to be oriented. The asemantic models would not expect a SNARC effect, because this kind of task does not require access to the number line. Finally, Dehaene's triple-code model entails both routes: 
Transcoding can be done either directly or via magnitude information. A SNARC effect would then point to the dominance of the indirect route over the direct route.

Thus, if a SNARC effect arises in an arabic-to-verbal transcoding task, all current models will need some revision. We used an experimental setting in which subjects had to press one of two response buttons to indicate whether the phoneme /e/ was present in the name of a visually presented arabic digit. Because Dehaene and collaborators were the first to report the spatialnumerical association of response codes, we decided first to replicate their findings in order to evaluate the robustness of the effect. A successful replication would allow us to interpret correctly the eventual absence of a SNARC-effect in the subsequent experiments

\section{EXPERIMENT 1}

Experiment 1, which matched as closely as possible Dehaene et al.'s Experiment 3 (1993), was crucial to ascertain whether it was relative magnitude that caused the SNARC effect, rather than some absolute stimulus characteristic. In one block, subjects had to judge parity for numbers in the range $0-5$; in another block, the numbers covered the interval 4-9. If the SNARC effect stems from absolute stimulus characteristics, no difference would be expected for the numbers 4 and 5 between both intervals. If, on the other hand, the SNARC effect is due to the magnitude of each stimulus relative to the range of numbers tested, than an interaction would be expected between interval and the association between number and response key. The numbers 4 and 5 are expected to be associated with a right-hand key for the interval 0 5 because they are positioned to the right of the mental number line, whereas they should be associated with the left-hand key in the interval 4-9 because they are located on the extreme left of the interval being tested.

\section{Method}

\section{Subjects}

Twenty-four native Dutch speaking subjects (16 female, 8 male), with an average age of 23.4 years, were tested; 18 were final-year undergraduate students in psychology, and 6 were members of the staff of the psychology department. None had a particular training in mathematics.

\section{Instructions}

Subjects were told to judge the parity of digits by pressing one of two response keys. They were explicitly instructed on the range of digits used in each block. The importance of both speed and accuracy was stressed. 


\section{Procedure}

All subjects participated in two blocks (one covering the interval $0-5$, the other 4-9), counterbalanced across subjects. Within each block, subjects were tested twice: once with the even response assigned to the right-hand key, once with the even response assigned to the left-hand key, in counterbalanced order. Each of the four sessions started with a training list of 12 items (all numbers being presented twice), and then each number was presented 15 times (in random order, with the restriction that each number followed every other number 3 times). This resulted in a total of 102 items per session. There was a short break between sessions.

The response board, with two response buttons separated $25 \mathrm{~mm}$ from one another, was connected to a PC-compatible computer (286-processor) with a black and white Hercules-type screen. Each trial started with an empty rectangular frame $(25 \mathrm{~mm} \times 33 \mathrm{~mm})$ presented in the centre of the screen for $300 \mathrm{msec}$. Thereafter the actual target number appeared $(15 \mathrm{~mm} \times 21 \mathrm{~mm})$ for a duration of $1300 \mathrm{msec}$, during which response time was measured with a precision of $1 \mathrm{msec}$. The frame and the number were then erased, and the screen remained blank for an interval of $1500 \mathrm{msec}$.

\section{Results}

Average error rate over subjects was $3.8 \%$ (with a maximum of $10 \%$ ), and there was no speed-accuracy trade-off, as indicated by a positive correlation between RT and number of errors over the 24 cells of the design, $r=+0.85$; $n=24, p<0.01$. Mean RTs of the correct responses for the numbers in the interval 0-5 were, respectively, $525,515,490,478,477$, and $481 \mathrm{msec}$. In the interval 4-9 the mean RTs were, respectively, 455, 477, 478, 464, 460, and 489 msec.

In a first analysis, the medians of the correct responses were analysed in a 2 (order of blocks) $\times 2$ (order within blocks) $\times 2$ (interval: 0-5 or 4-9) $\times 2$ parity: odd or even) $\times 3$ (magnitude: small, medium, or large in the interval tested) $\times 2$ (side of response: right or left) ANOVA, as in Dehaene et al. (1993). This yielded the same results as reported in Dehaene et al. In particular, the SNARC effect was present, as indicated by a significant Magnitude $\times$ Side of response interaction, $F(2,40)=14.54 M S_{\mathrm{e}}=1239.4$, $p<0.01$.

However, it seemed to us that another way of analysing the data would capture the essence of the SNARC effect more clearly. Because the SNARC effect stems from an association between the position of a number on the leftto-right-oriented number line and the side of response, it predicts a negative relation between number magnitude and the difference in RT between the right and left hand $(\mathrm{dRT})$. The most straightforward manner to confirm this 
relationship statistically is to regress $\mathrm{dRT}$ on number magnitude and to test the reliability of the regression slope.

This was done by means of a regression analysis of repeated-measures data described by Lorch and Myers (1990, Method 3). In a first step, for each subject the median RT of the correct responses was computed for each number, separately for left and right responses, and for the two intervals. On the basis of these medians, dRTs were computed by subtracting the median RT for left-hand responses from the median RT for right-hand responses. If there is an association between response side and number magnitude, we would expect a negative correlation between number magnitude and $\mathrm{dRT}$ : relatively small numbers should elicit faster left responses, resulting in positive dRTs, whereas relatively large numbers should elicit faster right responses, and thus negative dRTs. Therefore, in a second step, two regression equations were computed per subject (one for each interval) with number magnitude and practice as predictor variables. The latter variable was included because dRTs may also be influenced by the order in which subjects had to press the response buttons: If subjects first have to press the left button in response to even numbers, their left-hand responses may, overall, be slower than their righthand responses due to practice, while the reverse holds for subjects who have to respond to even numbers with their right hand first. Those numbers that were first responded to with the left hand were coded as -0.5 , those that were first responded to with the right hand were coded as +0.5 , in order not to influence the magnitude of the intercept. In a third step, $t$-tests were performed to test whether the regression weights of the group deviated significantly from zero and to test whether the slopes from the two intervals differed from one another.

There are several advantages to this analysis. First, the presence of a SNARC effect is judged by a main effect (does the slope of number magnitude differ from zero?), rather than by the presence of an interaction between magnitude and side of response. Second, the number magnitude factor is considered as a continuous variable. Third, the new analysis allows the quantification of the size of the effect (how large is the slope?), rather than a mere qualitative judgement of presence versus absence of the effect. Fourth, the effect of additional variables such as practice can easily be inserted and partialled out, which proved to be of importance in Experiment 3. Fifth, the method evaluates the linear relation between number size and difference in RT for each individual subject, reducing the chance of overestimating the SNARC effect due to group averaging. Finally, the method is more flexible. As will be shown in Experiments 2 and 3, the method does not require an orthogonal combination of the factors in the design. This is interesting when measures other than parity judgement are investigated, because not all variables allow a sequential alternation between number magnitude and response code, as is the case with the distinction between odd and even numbers. 

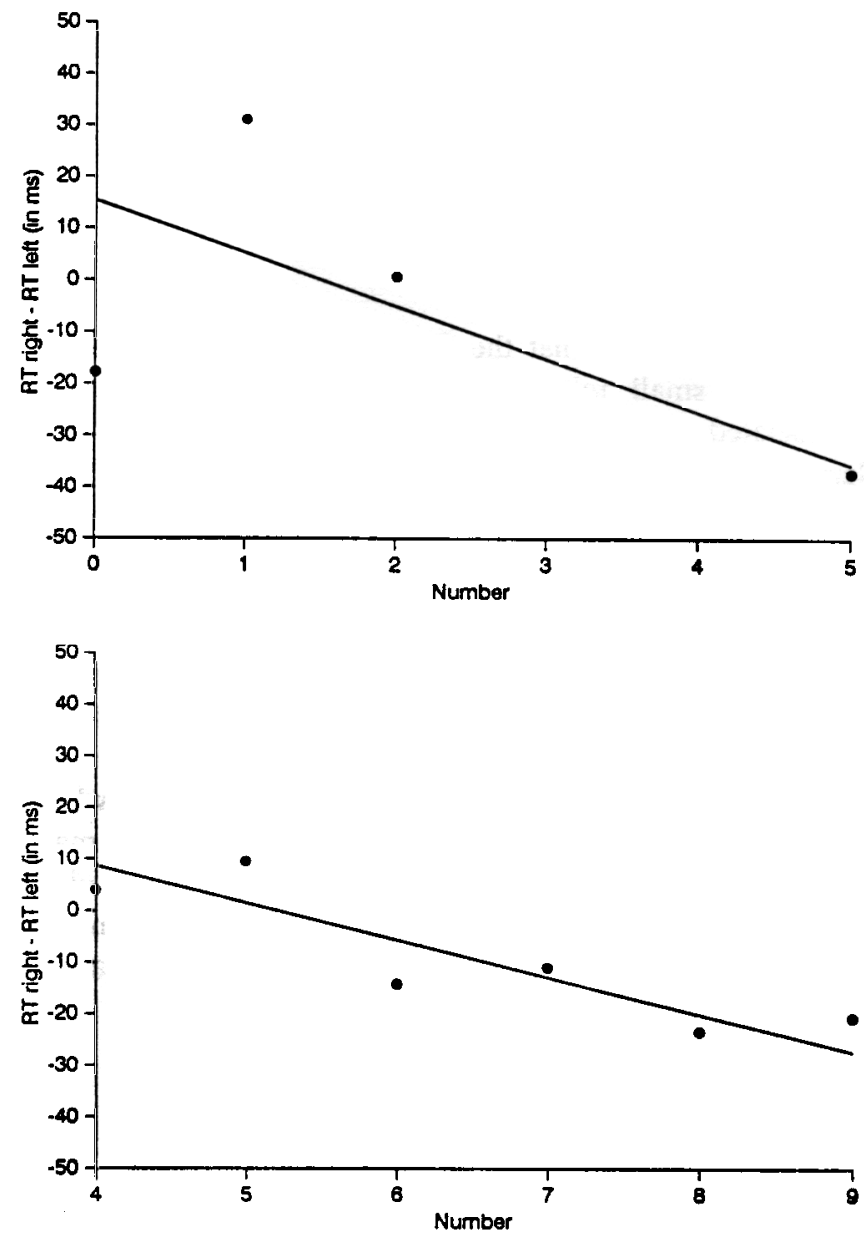

FIG. 1. Observed data and regression lines representing RT differences between right-hand and left-hand responses as a function of number magnitude in a parity judgement task. Positive differences indicate faster lefthand responses; negative differences indicate faster right-hand responses. Upper panel: interval 0-5; bottom panel: interval 4-9.

As can be seen in Fig. 1, the expected negative slope between number magnitude and $\mathrm{dRT}$ was present for both intervals. The regression lines are described by the following equations:

Interval 0-5: $\mathrm{dRT}=15.43-10.18$ (Magnitude) +8.47 (Practice)

Interval 4-9: $\mathrm{dRT}=37.58-7.19$ (Magnitude) +22.82 (Practice) 
The regression weights of magnitude deviate significantly from zero in both intervals: $t(23)=-3.31, S D=15.1, p<0.01$, and $t(23)=-3.13$, $S D=112, p<0.01$, respectively. The effect of practice did not reach significance in either of the two intervals. Finally, a dependent samples $t$-test showed that the slopes of the two intervals did not differ, $t(23)<1$.

\section{Discussion}

The pattern of results shows that the SNARC effect is present in our data: Numbers that were small relative to the interval of numbers used were preferably associated with a left-hand response, and numbers that were relatively large were associated with a right-hand response. In line with Dehaene et al. (1993), this is taken as evidence that (1) numbers are represented on a number line that is oriented from left to right, and (2) magnitude information is automatically and necessarily accessed during parity judgement.

There was one exception to the predicted pattern and to the results obtained in the original Dehaene et al. experiments: Responses to 0 were faster with the right hand. One explanation for this finding might be that 0 is processed differently from the other numbers. Almost all subjects asked whether 0 was to be considered as an even number or not. Another possibility may be that 0 is located not at the left but at the extreme right end of the number line. For instance, on telephones and keyboards, 0 follows 9 , rather than preceding 1 . Further testing is necessary, however, to determine the reliability of this exception.

\section{EXPERIMENT 2}

Now that the SNARC effect has proven to be a marker for the importance of magnitude information in numerical processing, this allows us to try to find experimental evidence for semantically mediated transcoding, as postulated by McCloskey et al. (1985, 1986, 1987), by Gallistel and Gelman (1992), and partly by Dehaene (1992b).

This was done with an experimental task that required transcoding from arabic input to verbal output. In order to combine such transcoding with a binary left/right response, subjects were asked to indicate whether an /e/ phoneme was present in the name of a visually presented arabic digit. If arabicto-verbal transcoding is semantically mediated, it may be expected that this response would be made faster with the left hand for smaller numbers and with the right hand for larger numbers. If transcoding is done asemantically, no SNARC effect would be expected. 


\section{Method}

\section{Subjects}

Twenty-three Dutch speaking subjects (12 female, 11 male) with an average age of 25.7 years were tested; 14 of the subjects were members of the staff of the psychology department, 5 were final-year students, and 4 were undergraduate students from diverse non-psychology departments. Three subjects were excluded from analyses because of their high error rate (more than 15\%). None of the subjects had participated in Experiment 1 or had knowledge of the purposes of the experiment.

\section{Instructions}

Subjects were told to judge whether there was an e-sound in the name of the number presented in the arabic modality, by pressing one of two response buttons. Both speed and accuracy were stressed in the instructions, and the interval of numbers was explicitly mentioned.

\section{Stimuli}

Unlike Experiment 1, only one interval of numbers was used: 0-9. The Dutch words for these numbers are "nul", "een", "twee", "drie", "vier", "vijf", "zes", "zeven", "acht", and "negen", with the /e/ phoneme present in 1, 2, 6, 7, and 9. It must be noted that the e-sound in 6 is short, whereas it is long in all other cases. This fact was explicitly mentioned in the instructions.

\section{Procedure}

Subjects participated in two blocks, one with the e-sound assigned to the left-hand button, and one with the e-sound assigned to the right-hand button; the order counterbalanced across subjects. Each block started with a training session in which all numbers were presented once. In each test block, the numbers were presented nine times, in randomised order with the restriction that each number followed each of the other numbers once. This resulted in 90 items for each block, with a short resting period between blocks. The apparatus and the presentation details were the same as in Experiment 1.

\section{Results}

The average rate was $5 \%$, and it did not exceed $9.4 \%$ per subject. A speedaccuracy trade-off was absent, as indicated by a significantly positive correlation between RT and number of errors, computed over 20 data couples 
(10 numbers separated for left and right responses), $r=0.67, n=20, p<0.01$. Mean RTs for the correct responses to the digits 0 to 9 were, respectively, 592; $569,558,650,624,591,613,568,605$, and $540 \mathrm{msec}$.

Regression weights were computed as in Experiment 1, except that there was only one interval, $0-9$. This resulted in the equation:

$$
\mathrm{dRT}=22.81-6.01 \text { (Magnitude) }+ \text { 89.32(Practice) }
$$

(see Fig. 2). The magnitude coefficient differed significantly from zero, $t(19)=-2.95, S D=9.1, p<0.01$, as did the practice predictor, $t(19)=4.19$; $S D=95.3, p<0.01$.

\section{Discussion}

The results of Experiment 2 clearly show the presence of a SNARC effect in a task in which subjects were required to translate a visually presented arabic number into its verbal representation in order to give a response. In line with Dehaene et al. (1993), this is interpreted as evidence for the fact that number magnitude, being represented on an analogue, left-to-right-oriented number line, is automatically addressed in a task in which this magnitude information is irrelevant for generating a correct response.

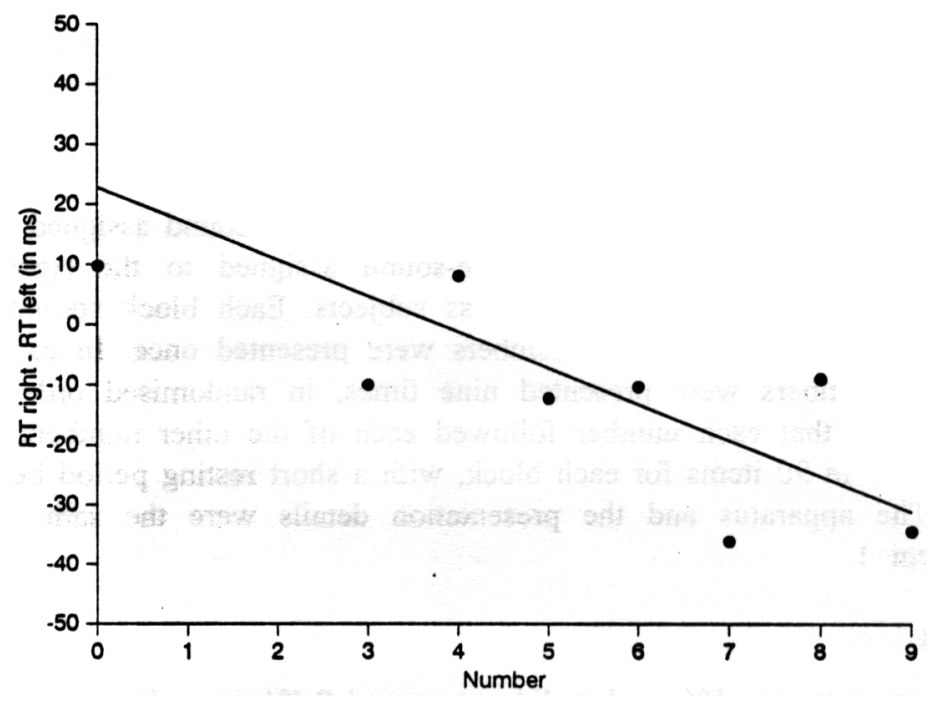

FIG. 2. Observed data and regression lines representing $R T$ differences between right-hand and left-hand responses as a function of number magnitude in a phonememonitoring task. Positive differences indicate faster left-hand responses; negative differences indicate faster right-hand responses. 
The influence of magnitude information in the present transcoding task is in agreement with the lay-out of the numerical system as hypothesised by McCloskey (1992) and by Gallistel and Gelman (1992). All numerical input, regardless of its format and regardless of task requirements, is automatically and necessarily translated to an abstract code, representing the number's magnitude. As to the nature of this abstract magnitude code, however, McCloskey's base-ten representation cannot explain in a parsimonious way the association between number magnitude and the spatial left-right coordinates. A representation on an analogue, left-to-right-oriented number line is more plausible.

On the other hand, the pattern of results causes problems for the cognitive architecture of Noël and Seron's (1993) preferred entry code model, because it locates magnitude information outside the arabic-to-verbal or verbal-to-arabic transcoding pathway and predicts no influence of number magnitude for this particular task.

As for Dehaene's triple-code model (1992b), the results are not incompatible, but at least they point to the dominance of the indirect semantic transcoding route over the direct asemantic transcoding route between the arabic and the verbal system.

There is one aspect of our data, however, that could cast doubts on the above interpretation. The practice effect (which was present in Experiment 2, but not in Experiment 1) could indicate the presence of a task-specific interference in the direct arabic-to-verbal transcoding route due to inexperience with the task and/or to particularities of the graphemic representation. The latter may be relevant because in Dutch the numbers 3 and 4 have the letter $e$ in their graphemic code ("drie" and "vier"), although it is not pronounced as an esound. The mean RTs show indeed that the numbers 3 and 4 elicited slow responses. Thus, it is possible that the arabic-to-verbal transcoding is done directly, but that graphemic interference causes a delay that gives the automatically activated magnitude information the opportunity to affect performance. This will be tested in the next experiment.

\section{EXPERIMENT 3}

In Experiment 3 we explicitly controlled for the influence of practice. If the direct arabic-to-verbal route is indeed the dominant one but is slowed down due to task-specific reasons, we can expect this route to regain its dominance if subjects become better practised in the task. As a consequence, the impact of the magnitude addressing route may be expected to decrease, resulting in a decrease of the SNARC effect. If, on the other hand, the SNARC effect does not change with practice, we would have evidence not only that magnitude information is automatically activated, but that this indirect route dominates the direct route. 


\section{Method}

\section{Subjects}

Twenty-six Dutch speaking subjects (17 female, 9 male) participated in the experiment, all first-year undergraduate students in psychology, with an average age of 20.8 years. For all of them it was the first time they had participated in an experiment.

\section{Instructions and Stimuli}

These were the same as in Experiment 2.

\section{Procedure}

Subjects participated in two sessions, one with the /e/-phoneme assigned to the left-hand button, one with the /e/-phoneme assigned to the right-hand button, the order counter-balanced across subjects. Each of these sessions was preceded by a training session in which each number was presented once. Practice was controlled by further dividing each session into 3 blocks. This resulted in a total number of 6 blocks, with a change of side of response after the first 3 blocks. Another possibility would have been to change the side of response for each block. However, we preferred the first option because it would be too confusing for subjects to change the side of response repeatedly, and this confusion could reduce the chances for the direct route to regain its influence. In every block, each number was presented 18 times, in pseudorandom order with the restriction that each number followed each other number twice. This resulted in 180 trials for each block. Between each block there was a short resting period. The apparatus and the presentation details were the same as in Experiments 1 and 2.

\section{Results}

The error rate did not exceed $10.8 \%$ per subject (average $=3.7 \%$ ). There was no speed-accuracy trade-off, as indicated by the correlation between RTs and numbers of errors computed over the 60 cells of the design ( 3 Blocks $\times 10$ Numbers $\times$ Left or Right response). The correlation was positive, $r=0.61$, $n=60, p<0.01$. RTs of the correct responses averaged over sessions, blocks, and side of response were $536,539,550,588,572,540,545,536,560$, and $514 \mathrm{msec}$ for the digits in ascending order, respectively.

The dRTs were computed by comparing the blocks of the first session with the blocks of the second session (because the side of response was manipulated between sessions). Thus, the dRTs of the first block were computed by comparing the median RTs of the first block of the first session with the median RTs of the first block of the second session, and so on. Regression equations were computed with magnitude and practice as predictors. Practice 
was again coded as -0.5 or +0.5 depending on the hand that subjects started with in response to the number in question. The equations for the three blocks were, respectively,

$$
\begin{array}{ll}
\text { Block 1: } & \mathrm{dRT}=12.22-4.33 \text { (Magnitude) }+51.59 \text { (Practice) } \\
\text { Block 2: } & \mathrm{dRT}=6.21-4.33 \text { (Magnitude) }+51.59 \text { (Practice) } \\
\text { Block 3: } & \mathrm{dRT}=7.58-5.03 \text { (Magnitude) }+43.47 \text { (Practice) }
\end{array}
$$

(see Fig. 3). Magnitude was significant in all three blocks-Block $1, S D=7.8$, $t(25)=-2.85, p<0.01$; Block 2, $S D=5.3, t(25)=-4.15, p<0.01$; Block 3, $S D=5.33, t(25)=-4.81, p<0.01$-as was Practice-Block $1, S D=98.0$, $t(25)=6.52, p<0.01$; Block 2, $S D=92.7, t(25)=2.84, p<0.01$; Block 3, $S D=70.7, t(25)=2.13, p<0.01$.

An ANOVA with repeated measures on the magnitude coefficients, furthermore, showed no difference between the three blocks, $F<1$, indicating that the SNARC effect stayed the same across blocks. The influence of practice varied significantly between blocks, $F(2,50)=20.61, M S_{\mathrm{e}}=2565.8, p<0.001$; Tukey comparisons showed that this was due to a decrease from the first to the second block.

\section{Discussion}

Experiment 3 shows that the SNARC effect in Experiment 2 remains if the effect due to practice is diminished considerably. This indicates that there is no reason to believe that the dominance of the direct route, without access to magnitude information, was obscured in Experiment 2 by inexperience with the task or the specific stimulus characteristics. There is no indication that the slope of the SNARC effect decreases with practice. This provides us with further evidence that the indirect, number-line-addressing pathway in Dehaene's triple-code model is the dominant route, if not the only one.

\section{GENERAL DISCUSSION}

Models of numerical cognition differ with respect to the necessity of a central semantic system that mediates all number processing. They also differ in the nature of this system. In the present paper we evaluated the different approaches by looking at the recently discovered Spatial-Numerical Association of Response Codes (the SNARC effect: Dehaene et al., 1993), which has been interpreted as an indication of access to an oriented semantic number line.

In Experiment 1, we replicated Dehaene et al. (1993) and showed that in a parity judgement task responses to small numbers are faster with the left hand whereas responses to large numbers are faster with the right hand. In two subsequent experiments the SNARC effect was extended to a phonememonitoring task that required arabic-to-verbal transcoding. Similar extensions 

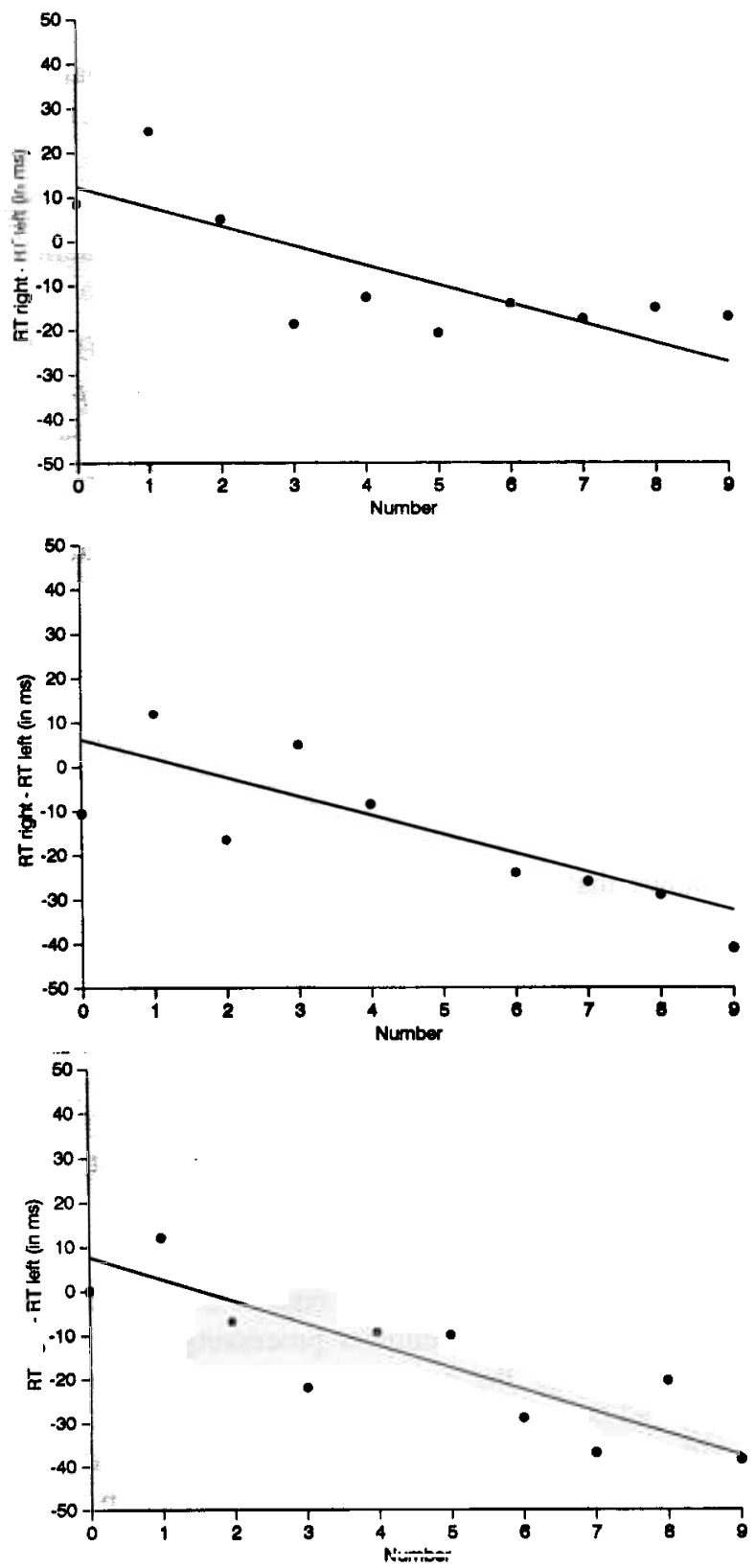

FIG. 3, Observed data and regression lines representing RT differences between right-hand and lefthand responses as a tunetion oi number magnitude in a phonemo-monitoring task at three levels of practice. Positive differences indicate faster left-hand responses; negative differences indicate faster right-hand responses. Upper panel: first level of practice; middle panel: second level of practice; bottom panel: third level of practice. 
of the SNARC effect to non-numerical tasks (vowel-consonant and symmetrical-asymmetrical judgement) have been reported recently by Huha, Berch, and Krikorian (1995).

If the SNARC effect really is an indication of the fact that the semantic number magnitude system has been addressed as hypothesised by Dehaene et al. (1993), then our findings have implications for several existing models.

Noël and Seron's (1993) preferred entry code model in its current form cannot account for the data because it does not position magnitude information inside the pathway between arabic and verbal representation stores. This does not preclude, of course, the possibility that there may be individual differences in the preferred use of one or the other codes.

The general architecture of McCloskey et al's (1985) model is in agreement with our data, except for the fact that the base-ten representation is difficult to reconcile with the SNARC effect. An oriented number line seems to account better for this spatial-numerical association. The orientedness should also be added explicitly to Gallistel and Gelman's model (1992).

Finally, Dehaene's triple-code model (1992b) agrees with our results if one accepts that in normal subjects the indirect transcoding route that involves the semantic number system dominates the direct transcoding route that does not involve this system.

Irrespective of which model is easiest to adapt, our data clearly show that number magnitude is more important than is usually assumed. Even in a task as simple as phoneme monitoring in digit names, number magnitude plays a role.

Manuscript received 7 April 1995

Revised manuscript received 28 September 1995

\section{REFERENCES}

Campbell, J.I.D. (1994). Architectures for numerical cognition. Cognition, 53, 1-44.

Campbell, J.I.D., \& Clark, J.M. (1988). An encoding-complex view of cognitive number processing: Comment on McCloskey, Sokol, \& Goodman (1986). Journal of Experimental Psychology: General, 117, 204-214.

Dehaene, S. (Ed.) (1992a). Numerical Cognition: Special issue. Cognition, 44, 1-196.

Dehaene, S. (1992b). Varieties of numerical abilities. Cognition, 44, 1-42.

Dehaene, S., \& Akhavein, R. (1995). Attention, automaticity, and levels of representation in number processing. Journal of Experimental Psychology: Learning, Memory and Cognition, 21, 314326.

Dehaene, S., Bossini, S., \& Giraux, P. (1993). The mental representation of parity and number magnitude. Journal of Experimental Psychology: General, 122, 371-396.

Gallistel, C.R., \& Gelman, R. (1992). Preverbal and verbal counting and computation. Cognition, 44, 43-74.

Henik, A., \& Tzelgov, J. (1982). Is three greater than five: The relation between physical and semantic size in comparison tasks. Memory and Cognition, 10, 389-395. 
Huha, E., Berch, D., \& Krikorian, R. (1995). Obligatory activation of magnitude information during non-numerical judgments of Arabic numerals. Paper presented at a meeting of the American Psychological Society, New York, 29 June-2 July.

Lorch, R.F., Jr., \& Myers, J.L. (1990). Regression analyses of repeated measures data in cognition research. Journal of Experimental Psychology: Learning, Memory, \& Cognition, 16, 149-157.

McCloskey, M. (1992). Cognition mechanisms in numerical processing: Evidence from acquired dyscalculia. Cognition, 44, 107-157.

McCloskey, M., \& Caramazza, A. (1987). Cognitive mechanisms in normal and impaired number processing. In G. Deloche \& X. Seron (Eds.), Mathematical disabilities: A cognitive neuropsychological perspective (pp. 201-219). Hillsdale, NJ: Lawrence Erlbaum Associates, Inc.

McCloskey, M., Caramazza, A., \& Basili, A. (1985). Cognitive mechanisms in number processing and calculation: Evidence from dyscalculia. Brain and Cognition, 4, 171-196.

McCloskey, M., Sokol, S.M., \& Goodman, R.A. (1986). Cognitive processes in verbal-number production: Inferences from the performance of brain-damaged subjects. Journal of Experimental Psychology: General, 115, 307-330.

Noël, M., \& Seron, X. (1993). Arabic number reading deficit: A single case study or when 236 is read (2306) and judged superior to 1258. Cognitive Neuropsychology, 10, 317-339. 